University of Wollongong

Research Online

Faculty of Informatics - Papers (Archive)

Faculty of Engineering and Information

Sciences

May 2001

\title{
Perfect Reconstruction Integer-modulated Filter Banks
}

Alfred Mertins

University of Wollongong, mertins@uow.edu.au

T. Karp

Texas Tech University

Follow this and additional works at: https://ro.uow.edu.au/infopapers

Part of the Physical Sciences and Mathematics Commons

\section{Recommended Citation}

Mertins, Alfred and Karp, T.: Perfect Reconstruction Integer-modulated Filter Banks 2001.

https://ro.uow.edu.au/infopapers/16

Research Online is the open access institutional repository for the University of Wollongong. For further information contact the UOW Library: research-pubs@uow.edu.au 


\title{
Perfect Reconstruction Integer-modulated Filter Banks
}

\author{
Abstract \\ In this paper, we present design methods for perfect reconstruction (PR) integer-modulated filter banks, \\ including biorthogonal (low-delay) filter banks. Both the prototype filter and the modulation sequences are \\ composed of integers, thus allowing,efficient hardware implementations. To derive such filter banks, we \\ first extend the PR conditions known for cosine modulation to other, more general, modulation schemes. \\ We present solutions where the PR conditions on the prototype and the modulation are entirely decoupled \\ and where some simple coupling is introduced. The conditions are derived for both even and odd \\ numbers of channels. Design examples are presented for both cases.

\section{Keywords} \\ filters, modulation, signal reconstruction \\ Disciplines \\ Physical Sciences and Mathematics

\section{Publication Details} \\ This article was originally published as: Mertins, A \& Karp, T, Perfect Reconstruction Integer-modulated \\ Filter Banks, IEEE International Conference on Acoustics, Speech, and Signal Processing, Proceedings, \\ (ICASSP '01), 7-11 May 2001, 6, 3605-3608. Copyright IEEE 2001.
}




\section{PERFECT RECONSTRUCTION INTEGER-MODULATED FILTER BANKS}

\author{
Alfred Mertins \\ University of Wollongong \\ School of Elec., Comp., and Tele. Engineering \\ Wollongong, NSW 2522, Australia \\ Email: mertins@uow.edu.au
}

\author{
Tanja Karp \\ Texas Tech University \\ Department of Electrical and Computer Engineering \\ Box 43102, Lubbock, TX 79409-3102, USA \\ Email: tanja.karp@ttu.edu
}

\begin{abstract}
In this paper, we present design methods for perfect reconstruction (PR) integer-modulated filter banks, including biorthogonal (low-delay) filter banks. Both the prototype filter and the modulation sequences are composed of integers, thus allowing, efficient hardware implementations. To derive such filter banks, we first extend the PR conditions known for cosine modulation to other, more general, modulation schemes. We present solutions where the PR conditions on the prototype and the modulation are entirely decoupled and where some simple coupling is introduced. The conditions are derived for both even and odd numbers of channels. Design examples are presented for both cases.
\end{abstract}

\section{INTRODUCTION}

To implement a filter bank on a processor with finiteprecision arithmetic, one usually needs to quantize the filter coefficients. This, however, results in loss of the perfect reconstruction (PR) property. It is therefore of significant interest to design filter banks directly in such a way that PR can be achieved with integer arithmetic. Integer-coefficient prototypes for paraunitary filter banks were designed in [1]. Design methods for PR filter banks with integer modulation and integer prototypes have been presented in [2-5]. In [2] the integer modulation sequences are designed on the basis of the dyadic symmetry principle [6], and the filter lengths, $L$, are restricted to the case $L=2 M$, where $M$ is the number of bands. In $[3,4]$ prototypes with lengths $L \geq 2 M$ and even $M$ are considered. The work in [5] considers the sumof-powers-of-two implementation of the prototype and the cosine modulation. Further design methods for integer DCT matrices suitable for the use in integer filter banks have been presented in [7]. Thus far, all integer solutions presented in the literature consider an even number of channels. In this paper, we generalize the methods from [4] to an arbitrary number of channels (odd and even). We present solutions where the PR conditions on the prototype and the modulation are entirely decoupled and where some simple coupling is introduced. In the coupled case, the requirements on the prototype and the modulation sequences can be traded off between the two, resulting in an increased design freedom compared to the decoupled case.

\section{PR CONDITIONS FOR MODULATED FILTER BANKS}

In this section, we present the PR conditions for modulated filter banks with real-valued modulation sequences. We start by looking at cosine modulation and then generalize the conditions to other, more general modulation sequences. We consider critical subsampling and the case where the same FIR prototype is used on the analysis and synthesis sides. The number of channels $(M)$ may be even or odd. The overall delay $(D)$ of the analysis/synthesis system is assumed to be of the form

$$
D=2 s M+2 M-1
$$

with $s$ being an integer.

The analysis and synthesis filters, $h_{k}(n)$ and $g_{k}(n), k=$ $0, \ldots, M-1$ are derived from a length- $L$ prototype filter $p(n)$ as

$$
h_{k}(n)=p(n) t_{1, k}(n), \quad g_{k}(n)=p(n) t_{2, k}(n)
$$

for $n=0,1, \ldots, L-1$. The sequences $t_{1, k}(n)$ and $t_{2, k}(n)$ provide the modulation.

\subsection{PR Conditions for Cosine Modulation}

For cosine modulation the sequences $t_{1, k}(n)$ and $t_{2, k}(n)$ are considered to be given by

$$
\begin{gathered}
t_{1, k}(n)=2 \cos \left[\frac{\pi}{M}\left(k+\frac{1}{2}\right)\left(n-\frac{D}{2}\right)+\phi_{k}\right], \\
t_{2, k}(n)=2 \cos \left[\frac{\pi}{M}\left(k+\frac{1}{2}\right)\left(n-\frac{D}{2}\right)-\phi_{k}\right], \\
n=0, \ldots, L-1, k=0, \ldots, M-1, \phi_{k}=(-1)^{k} \pi / 4 .
\end{gathered}
$$


To derive the PR conditions, the polyphase matrices $\boldsymbol{E}(z)$ and $\boldsymbol{R}(z)$ of the analysis and synthesis filter banks are introduced as

$$
\begin{aligned}
\boldsymbol{E}(z) & =\boldsymbol{T}_{1}\left[\begin{array}{c}
\boldsymbol{P}_{0}\left(z^{2}\right) \\
z^{-1} \boldsymbol{P}_{1}\left(z^{2}\right)
\end{array}\right], \\
\boldsymbol{R}(z) & =\left[z^{-1} \boldsymbol{Q}_{1}\left(z^{2}\right), \quad \boldsymbol{Q}_{0}\left(z^{2}\right)\right] \boldsymbol{T}_{2}^{T} .
\end{aligned}
$$

The $M \times 2 M$ modulation matrices $\boldsymbol{T}_{1}$ and $\boldsymbol{T}_{2}$ in (5) and (6) are given by

$$
\begin{aligned}
{\left[\boldsymbol{T}_{1}\right]_{k, n} } & =t_{1, k}(n) \\
{\left[\boldsymbol{T}_{2}\right]_{k, 2 M-1-n} } & =t_{2, k}(n)
\end{aligned}
$$

where $k=0,1, \ldots, M-1$ and $n=0,1, \ldots, 2 M-1$. The matrices $\boldsymbol{P}_{0}\left(z^{2}\right), \boldsymbol{P}_{1}\left(z^{2}\right), \boldsymbol{Q}_{0}\left(z^{2}\right), \boldsymbol{Q}_{1}\left(z^{2}\right)$ are defined as

$$
\begin{aligned}
& \boldsymbol{P}_{0}\left(z^{2}\right)=\operatorname{diag}\left[P_{0}\left(-z^{2}\right), \ldots, P_{M-1}\left(-z^{2}\right)\right], \\
& \boldsymbol{Q}_{0}\left(z^{2}\right)=\operatorname{diag}\left[P_{M-1}\left(-z^{2}\right), \ldots, P_{0}\left(-z^{2}\right)\right], \\
& \boldsymbol{P}_{1}\left(z^{2}\right)=\operatorname{diag}\left[P_{M}\left(-z^{2}\right), \ldots, P_{2 M-1}\left(-z^{2}\right)\right], \\
& \boldsymbol{Q}_{1}\left(z^{2}\right)=\operatorname{diag}\left[P_{2 M-1}\left(-z^{2}\right), \ldots, P_{M}\left(-z^{2}\right)\right]
\end{aligned}
$$

with $P_{j}(z)$ being the type-1 polyphase components of the prototype: $P_{j}(z)=\sum_{\ell} p(2 \ell M+j) z^{-\ell}, j=0, \ldots, 2 M-1$.

For the cosine modulation (3) it turns out that

$$
\boldsymbol{T}_{2}^{\mathrm{T}} \boldsymbol{T}_{1}=2 M\left[\begin{array}{cc}
(-1)^{s} \boldsymbol{I}_{M}+\boldsymbol{J}_{M} & \mathbf{0} \\
\mathbf{0} & (-1)^{s} \boldsymbol{I}_{M}-\boldsymbol{J}_{M}
\end{array}\right]
$$

where $\boldsymbol{I}_{M}$ and $\boldsymbol{J}_{M}$ are the $M \times M$ identity and counter identity matrices, respectively.

The PR conditions for a reconstruction of an input signal with a delay $D=2 s M+2 M-1$ can be formulated in the polyphase domain as

$$
\boldsymbol{R}(z) \boldsymbol{E}(z)=z^{-2 s-1} \boldsymbol{I}_{M}
$$

Inserting (5) and (6) into (10) and simplifying the expression obtained using (9) yields

$$
\left[z^{-1} \boldsymbol{Q}_{1}\left(z^{2}\right) \boldsymbol{Q}_{0}\left(z^{2}\right)\right]\left[\begin{array}{c}
\boldsymbol{P}_{0}\left(z^{2}\right) \\
z^{-1} \boldsymbol{P}_{1}\left(z^{2}\right)
\end{array}\right]=z^{-2 s-1} \frac{(-1)^{s}}{2 M} \boldsymbol{I}_{M}
$$

After substitution of $z$ for $-z^{2}$ the conditions in (11) become

$$
P_{2 M-1-k}(z) P_{k}(z)+P_{M+k}(z) P_{M-1-k}(z)=\frac{1}{2 M} z^{-s}
$$

for $k=0, \ldots,\left\lceil\frac{M}{2}\right\rceil-1$, where \lceil\rceil denotes the ceiling operation. Note that (12) shows the PR conditions on the prototype for critically sampled, biorthogonal, cosine-modulated filter banks with the delay in (1). In the special case of paraunitary filter banks, a linear phase (symmetric) prototype is required. In this case, Eq. (12) leads to the following conditions on paraunitary prototypes:

$$
P_{k}(z) P_{k}\left(z^{-1}\right)+P_{M+k}(z) P_{M+k}\left(z^{-1}\right)=\frac{1}{2 M}
$$

for $k=0, \ldots,\left\lceil\frac{M}{2}\right\rceil-1$. Note that, given the delay in (1), the length of the paraunitary prototype is $L=2(s+1) M$.

\subsection{Generalized, Decoupled PR Conditions}

We now generalize the PR conditions from Section 2.1 to non-cosine modulation. For this we demand that

$$
\boldsymbol{T}_{2}^{\mathrm{T}} \boldsymbol{T}_{1}=\varepsilon\left[\begin{array}{cc}
(-1)^{s} \boldsymbol{I}_{M}+\boldsymbol{J}_{M} & \mathbf{0} \\
\mathbf{0} & (-1)^{s} \boldsymbol{I}_{M}-\boldsymbol{J}_{M}
\end{array}\right]
$$

with some factor $\varepsilon$. Thus, up to a scale factor, the same overall properties are obtained as with the original cosine modulation. Consequently, the PR conditions on the prototype and the modulation remain completely decoupled. By relaxing the condition (10) to perfect reconstruction up to a scale factor $\gamma$, which is required due to integer arithmetic,

$$
\boldsymbol{R}(z) \boldsymbol{E}(z)=z^{-2 s-1} \gamma \boldsymbol{I}_{M},
$$

the conditions (12) on the prototype become

$$
P_{2 M-1-k}(z) P_{k}(z)+P_{M+k}(z) P_{M-1-k}(z)=\frac{\gamma}{\varepsilon} z^{-s}
$$

for $k=0, \ldots,\left\lceil\frac{M}{2}\right\rceil-1$.

We now parameterize the matrices $\boldsymbol{T}_{1}$ and $\boldsymbol{T}_{2}$ as

$$
T_{1}=V_{1} Y_{1}, \quad T_{2}=V_{2} Y_{2}
$$

where the purpose of the $M \times 2 M$ matrices $Y_{1}$ and $Y_{2}$ is to periodically repeat the columns of the $M \times M$ matrices $V_{1}$ and $V_{2}$. To describe the scheme for all cases, we will have to distinguish between even and odd $M$ and $s$.

Even $M$ and arbitrary $s$. For even $M$ we define

$$
\begin{aligned}
& \boldsymbol{Y}_{1}=\left[\begin{array}{cccc}
(-1)^{s} \boldsymbol{J}_{M / 2} & \boldsymbol{I}_{M / 2} & \mathbf{0} & \mathbf{0} \\
\mathbf{0} & \mathbf{0} & \boldsymbol{I}_{M / 2} & -(-1)^{s} \boldsymbol{J}_{M / 2}
\end{array}\right] \\
& \boldsymbol{Y}_{2}=(-1)^{s} \boldsymbol{Y}_{1} .
\end{aligned}
$$

Provided that

$$
\boldsymbol{V}_{2}^{T} \boldsymbol{V}_{1}=\varepsilon \boldsymbol{I}_{M}
$$

matrices $\boldsymbol{T}_{1}$ and $\boldsymbol{T}_{2}$ according to (17) will satisfy (14)

Odd $M$ and even $s$. To describe $\boldsymbol{Y}_{1}$ and $\boldsymbol{Y}_{2}$ for odd $M$ it is convenient to express the matrices element wise. For $i=0, \ldots, M-1$ we define

$$
\left[\boldsymbol{Y}_{1}\right]_{i, k}= \begin{cases}\delta_{i, \mu-k}, & k=0, \ldots, \mu \\ \delta_{i, k-\mu}, & k=\mu+1, \ldots, M+\mu-1 \\ 0, & k=M+\mu \\ -\delta_{i, 2 M+\mu-k}, & k=M+\mu+1, \ldots, 2 M-1\end{cases}
$$




$$
\boldsymbol{Y}_{2}=\boldsymbol{Y}_{1}
$$

where $\mu=\left\lfloor\frac{M}{2}\right\rfloor$. The expression \lfloor\rfloor denotes the floor operation, and $\delta_{i, j}$ is the Kronecker symbol.

As can be easily verified, to satisfy (14), $V_{1}$ and $V_{2}$ need to satisfy

$$
\boldsymbol{V}_{2}^{T} V_{1}=\varepsilon \cdot \operatorname{diag}[2,1, \ldots, 1]
$$

Odd $M$ and odd $s$. We define

$$
\left[\boldsymbol{Y}_{1}\right]_{i, k}= \begin{cases}-\delta_{i, \mu-k-1}, & k=0, \ldots, \mu-1 \\ 0, & k=\mu \\ \delta_{i, k-\mu-1}, & k=\mu+1, \ldots, M+\mu \\ \delta_{i, 2 M+\mu-k-1}, & k=M+\mu+1, \ldots, 2 M-1\end{cases}
$$$$
\boldsymbol{Y}_{2}=-\boldsymbol{Y}_{1}
$$

for $i=0, \ldots, M-1$ with the same $\mu$ as for an even $s$. To satisfy (14), $V_{1}$ and $V_{2}$ now need to satisfy

$$
\boldsymbol{V}_{2}^{T} \boldsymbol{V}_{1}=\varepsilon \cdot \operatorname{diag}[1, \ldots, 1,2] .
$$

\subsection{Coupled Conditions}

In this section we show that it is possible to trade off some of the requirements on the prototype to the modulation sequences and vice versa. This gives us an increased design freedom when looking for integer solutions. We consider the formulation for $\boldsymbol{T}_{1}$ and $\boldsymbol{T}_{2}$ in (17) and relax the condition on $V_{1}$ and $V_{2}$ to

$$
\boldsymbol{V}_{2}^{T} \boldsymbol{V}_{1}=\boldsymbol{\Gamma}
$$

where $\boldsymbol{\Gamma}$ is a diagonal matrix with non-zero diagonal entries. To further specify $\Gamma$ we need to distinguish between even and odd $M$

Even $M$. For even $M, \Gamma$ is chosen as

$$
\Gamma=\operatorname{diag}\left[w_{\frac{M}{2}-1}, \ldots, w_{0}, w_{0}, \ldots, w_{\frac{M}{2}-1}\right] \text {. }
$$

The product $\boldsymbol{T}_{2}^{\boldsymbol{T}} \boldsymbol{T}_{1}$ then takes on the form

$$
\boldsymbol{T}_{2}^{T} \boldsymbol{T}_{1}=\left[\begin{array}{cc}
\Delta\left[(-1)^{s} \boldsymbol{I}+\boldsymbol{J}\right] & \mathbf{0} \\
\mathbf{0} & \Delta\left[(-1)^{s} \boldsymbol{I}-\boldsymbol{J}\right]
\end{array}\right]
$$

with

$$
\Delta=\operatorname{diag}\left[w_{0}, \ldots, w_{\frac{M}{2}-1}, w_{\frac{M}{2}-1}, \ldots, w_{0}\right] .
$$

Following the same derivation as in Section 2.1, we obtain the following PR conditions on the prototype:

$$
w_{k}\left[P_{k}(z) P_{2 M-1-k}(z)+P_{M+k}(z) P_{M-1-k}(z)\right]=\gamma z^{-s}
$$

Odd $M$ and even $s$. For this case we choose

$$
\Gamma=\operatorname{diag}\left[2 w_{\left\lfloor\frac{M}{2}\right\rfloor}, w_{\left\lfloor\frac{M}{2}\right\rfloor-1}, \ldots, w_{0}, w_{0}, \ldots, w_{\left\lfloor\frac{M}{2}\right\rfloor-1}\right]_{(28)}
$$

Again, the product $\boldsymbol{T}_{2}^{T} \boldsymbol{T}_{1}$ takes on the form (25), but now with

$$
\Delta=\operatorname{diag}\left[w_{0}, \ldots, w_{\left\lfloor\frac{M}{2}\right\rfloor-1}, w_{\left\lfloor\frac{M}{2}\right\rfloor}, \stackrel{\mathrm{w}}{\left\lfloor\frac{M}{2}\right\rfloor-1}, \ldots, w_{0}\right]
$$

The PR conditions on the prototype remain the same as in (27).

Odd $M$ and odd $s$. We choose

$$
\mathbf{\Gamma}=\operatorname{diag}\left[w_{\left\lfloor\frac{M}{2}\right\rfloor-1}, \ldots, w_{0}, w_{0}, \ldots, w_{\left\lfloor\frac{M}{2}\right\rfloor-1}, 2 w_{\left\lfloor\frac{M}{2}\right\rfloor}\right] \text {. }
$$

The product $\boldsymbol{T}_{2}^{T} \boldsymbol{T}_{1}$ takes on the form (25) with $\boldsymbol{\Delta}$ as in (29). The PR conditions on the prototype remain the same as in (27).

\section{DESIGN EXAMPLES}

The first example presents a low-delay filter bank with $M=8$ channels, a filter length of $L=32$, and an overall system delay of $D=15$ samples. By using a lifting structure, optimizing, and finally quantizing the the lifting coefficients, the following prototype satisfying (16) has been found:

$p(n)=\{50,60,80,80,108,116,118,120,150,140,120,120$, $88,76,58,40,10,10,0,0,0,0,3,4,-10,-10,0,0,0,0,3,4\}$.

Because of limited space, the lifting-based design procedure cannot be presented here in great detail. A complete description is given in [8].

An $8 \times 8$ integer matrix which resembles the symmetries found in the cosine matrix $V_{1}^{c}$ is found as [4]

$$
\boldsymbol{V}=\left[\begin{array}{rrrrrrrr}
27 & 28 & 24 & 23 & 19 & 14 & 9 & 5 \\
-28 & -19 & -5 & 14 & 24 & 27 & 23 & 9 \\
-24 & -5 & 23 & 28 & 9 & -19 & -27 & -14 \\
23 & -14 & -28 & 5 & 27 & 9 & -24 & -19 \\
19 & -24 & -9 & 27 & -5 & -28 & 14 & 23 \\
-14 & 27 & -19 & -9 & 28 & -23 & -5 & 24 \\
-9 & 23 & -27 & 24 & -14 & -5 & 19 & -28 \\
5 & -9 & 14 & -19 & 23 & -24 & 28 & -27
\end{array}\right]
$$

The frequency responses of the resulting analysis filters are depicted in Figure 1(a). Note that the gains of the analysis filters have been adjusted so that the average maximum gain of all filters in a graph is $0 \mathrm{~dB}$.

In a second design example the number of subbands is chosen as $M=5$, the overall system delay is $D=9$ and 


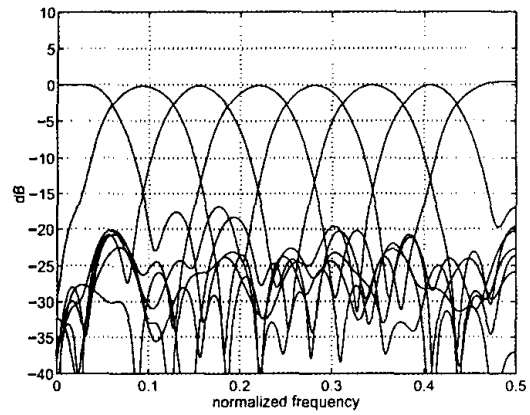

(a)

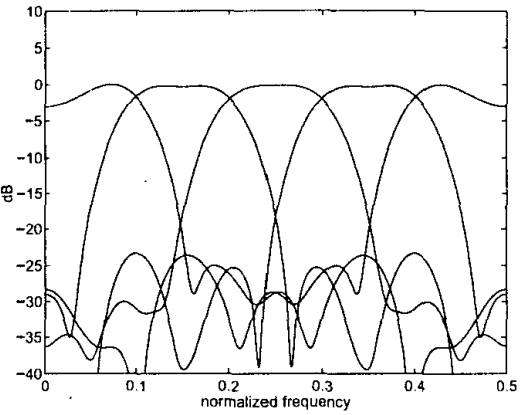

(b)

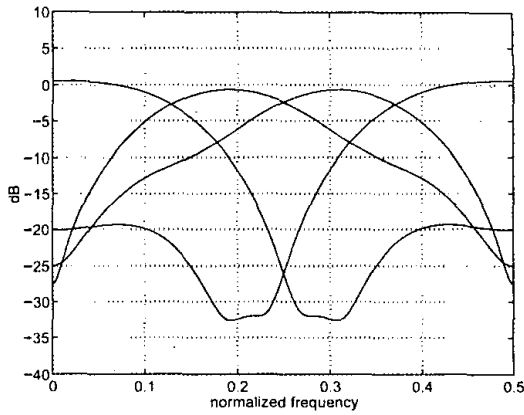

(c)

Figure 1: Normalized frequency response of low-delay integer-modulated filter banks. (a) $M=8$, uncoupled design; (b) $M=5$, uncoupled design; (c) $M=4$, coupled design.

the prototype filter length is $L=20$. A prototype satisfying (16) has been found as

$$
\begin{aligned}
p(n)= & \{10,20,30,40,50,52,53,49,41,34 \\
& 20,12,0,-4,-5,-8,-6,0,-2,-2\} .
\end{aligned}
$$

A suitable modulation is given by

$$
\boldsymbol{V}=\left[\begin{array}{rrrrr}
30 & 30 & 25 & 16 & 9 \\
-30 & -16 & 9 & 30 & 25 \\
-32 & 0 & 30 & 0 & -30 \\
30 & -16 & -9 & 30 & -25 \\
30 & -30 & 25 & -16 & 9
\end{array}\right]
$$

Note that all rows of $\boldsymbol{V}$ in (32), except the third one, resemble the symmetries found in the corresponding cosine matrix $\boldsymbol{V}_{1}^{c}$. The required matrices $\boldsymbol{V}_{1}$ and $\boldsymbol{V}_{2}$ are given by $\boldsymbol{V}_{1}=\boldsymbol{V}_{2}=\boldsymbol{V}$. The frequency responses of the resulting analysis filters for $M=5$ are depicted in Figure l(b).

A third example considers a four-channel filter bank with symmetric, paraunitary prototype of length $L=8$. A modulation matrix $\boldsymbol{V}$ has been found as

$$
\boldsymbol{V}=\left[\begin{array}{rrrr}
10 & 10 & 5 & 2 \\
-11 & 6 & 8 & 3 \\
-2 & 5 & -10 & 10 \\
-3 & 8 & -6 & -11
\end{array}\right]
$$

It satisfies $\boldsymbol{V}^{T} \boldsymbol{V}=\operatorname{diag}[234,225,225,234]$, which means that $w_{0}=225, w_{1}=234$. A suitable prototype is given by

$$
p(n)=\{1,3,4,5,5,4,3,1\}
$$

It is easy to see that (27) is satisfied with $\gamma=w_{0}\left(p^{2}(0)+\right.$ $\left.p^{2}(3)\right)=w_{1}\left(p^{2}(1)+p^{2}(2)\right)=5850$. The entire filter bank is paraunitary, although the matrix $\boldsymbol{V}$ is not orthogonal. The frequency responses of the resulting filters are depicted in Figure 1(c).

\section{CONCLUSIONS}

We have presented the PR conditions for non-cosine modulated filter banks, including paraunitary and biorthogonal, low-delay filter banks. It was shown that the generalized PR conditions can be utilized to design integer-modulated filter banks with perfect reconstruction and very good frequency selectivity. In the examples presented, the prototypes and modulation matrices contain fairly small integers, thus allowing efficient hardware implementation.

\section{REFERENCES}

[1] A. Mertins, "Subspace approach for the design of cosinemodulated filter banks with linear-phase prototype filter," IEEE Trans. Signal Processing, vol. 46, no. 10, pp. 2812 2818, Oct. 1998.

[2] M. Bi, S. H. Ong, and Y. H. Ang, "Integer-modulated FIR filter banks for image compression," IEEE Trans. Circ. and Syst. for Video Technology, vol. 8, no. 8, pp. 923-927, Dec. 1998.

[3] A. Mertins, T. Karp, and J. Kliewer, "Design of perfect reconstruction integer-modulated filter banks," in Proc. ISSPA '99, Brisbane, Qld, Australia, Aug. 1999, vol. 2, pp. 591-594.

[4] A. Mertins, T. Karp, and J. Kliewer, "Integer-modulated filter banks providing perfect reconstruction," in Proc. EUSIPCO, Tampere, Finland, Sept. 2000.

[5] S. C. Chan, W. Liu, and K. L. Ho, "Perfect reconstruction modulated filter banks with sum-of-powers-of-two coefficients," in Proc. 2000 IEEE International Symposium on Circuits and Systems, Geneva, Switzerland, May 2000, vol. 2, pp. 73-76.

[6] W. K. Cham, "Development of integer cosine transforms by the principle of dyadic symmetry," Proc. Inst. Elect. Eng., Part $I$, vol. 136, pp. 276-282, Aug. 1989.

[7] J. Liang and T. D. Tran, "Fast multiplierless approximation of the DCT with the lifting scheme," in Proc. SPIE Applications of Digital Image Processing XXIII, San Diego, CA, Aug. 2000.

[8] A. Mertins and T. Karp, "Modulated, perfect reconstruction filter banks with integer coefficients," IEEE Trans. Signal Processing, submitted 2001 . 